\title{
Preface
}

\section{Cognitive and Behavioral \\ Therapies for Insomnia: Who \\ Is It for? What's New? Where \\ Do We Go from Here?}

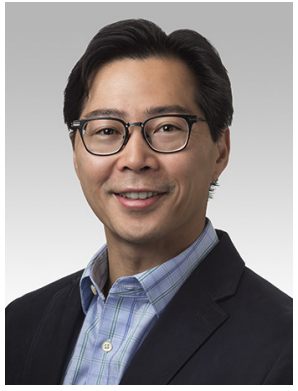

Jason C. Ong, PhD

Editor

Psychological and behavioral treatments have been used to treat insomnia for several decades. The first group of behavioral treatments used relaxation strategies, such as progressive muscle relaxation and biofeedback, to reduce physiologic arousal, which was thought to be the cause of insomnia. During the 1970s and 1980s, theorydriven approaches, including stimulus control, ${ }^{1}$ sleep hygiene, ${ }^{2}$ and sleep restriction therapy, ${ }^{3}$ were developed. As research began to implicate the role of cognitive factors (eg, maladaptive beliefs and attitudes about sleep, high effort to sleep) in chronic insomnia, ${ }^{4}$ techniques aimed at the cognitive level emerged. These individual techniques have been combined into a multicomponent treatment package that is known as cognitive behavior therapy for insomnia (CBT-I).

CBT-I is now recognized as the first-line treatment for chronic insomnia disorder. ${ }^{5}$ Despite this recognition, some controversies and unresolved questions remain. First, who should receive CBT-I? Is this a one-size-fits-all treatment package, and does it work the same for patients with various comorbid conditions? Second, are there alternatives to CBT-I? What is the evidence for these other nonpharmacologic approaches, and when should they be considered a treatment option? Third, how can we spread CBT-I to the masses? Although CBT-I has demonstrated evidence for efficacy, it remains massively underutilized relative to the high prevalence of insomnia. What are the barriers, and how do we address this problem?

This issue of Sleep Medicine Clinics addresses these questions by providing reviews on several key topics related to cognitive and behavioral treatments for insomnia. The first five articles discuss the delivery of CBT-I to specific populations. Gradisar and colleagues provide an overview of CBT-I in children and adolescents, an area that has received surprisingly little attention. In the next article, Arendt and colleagues discuss considerations in the delivery of CBT-I in patients with comorbid medical and psychiatric conditions, since insomnia often occurs with other conditions. Given the common cooccurrence of insomnia and depression, Arsanow and Manber provide an indepth review of delivering CBT-I for adolescents and adults with comorbid depression. With emerging interest in sleep and women's health, Nowakoski and Meers provide an overview of CBT-I in women, describing factors associated with pregnancy, menstrual cycles, and menopause. Finally, Martin and colleagues discuss issues in delivering CBT-I to military personnel, who typically present with complex physical and mental issues.

The second section of this issue includes articles on new alternatives or adaptations of CBT-I. Mindfulness meditation is a popular alternative 
approach, and Garland and colleagues provide a review and meta-analysis of the randomized controlled trials conducted in this area. Gunn and colleagues provide an overview of Brief Behavior Therapy, a shorter and more portable version of CBT-I. Intensive Sleep Retraining (ISR) is a novel behavioral approach designed to rapidly decrease sleep onset latency in an intense laboratory protocol. Lack and colleagues describe the history and evidence for ISR and explain how technology is now being used to translate the ISR protocol outside of sleep laboratories.

The third section consists of articles related to controversies and considerations of CBT-I. Morin and colleagues discuss the issues with the use of hypnotics when delivering CBT-I. Although CBT-I is an established treatment for chronic insomnia, the management of acute insomnia has generated controversy. Jason Ellis addresses this interesting question and describes a one-shot approach to treating acute insomnia. Buenaver and colleagues discuss issues involved with delivering CBT-I in the real world, including who is an appropriate candidate, using quality measures, and adaptations of CBT-I. In the final article, Drerup and Ahmed-Jauregui provide an overview of on-line delivery of CBT-I, an exciting area where technology is being leveraged to address the limited accessibility to CBT-I providers.

I would like to thank all of the contributors for their collective expertise in bringing this issue together. I hope that this collection of articles will inform the reader of the state-of-the-science and inspire continued innovations and advances in the treatment of insomnia.

Jason C. Ong, PhD Department of Neurology Center for Circadian and Sleep Medicine Northwestern University Feinberg School of Medicine 710 North Lake Shore Drive

Room 1004

Chicago, IL 60611, USA

E-mail address:

jason.ong@northwestern.edu

\section{REFERENCES}

1. Bootzin RR. Stimulus control treatment for insomnia. Presented at the 80th Annual Convention of the American Psychological Association. Honolulu (HI), August, 1972.

2. Hauri PJ. Current concepts: the sleep disorders. Kalamazoo (MI): The Upjohn Company; 1977.

3. Spielman AJ, Sasky P, Thorpy MJ. Treatment of chronic insomnia by restriction of time in bed. Sleep 1987:10(1):45-56.

4. Morin CM. Insomnia: psychological assessment and management. New York: The Guilford Press; 1993.

5. Qaseem A, Kansagara D, Forciea MA, et al. Clinical Guidelines Committee of the American College of P. Management of chronic insomnia disorder in adults: a clinical practice guideline from the American College of Physicians. Ann Intern Med 2016;165(2):125-33. 